\title{
Remaking memories: Reconsolidation updates positively motivated spatial memory in rats
}

\author{
Bethany Jones, ${ }^{1}$ Elizabeth Bukoski, ${ }^{2}$ Lynn Nadel, ${ }^{2}$ and Jean-Marc Fellous ${ }^{2,3,4}$ \\ ${ }^{1}$ Graduate Interdisciplinary Program in Neuroscience; ${ }^{2}$ Department of Psychology, University of Arizona, Tucson, \\ Arizona 85724, USA; ${ }^{3}$ Program in Applied Mathematics, University of Arizona, Tucson, Arizona 85721-0089, USA
}

\begin{abstract}
There is strong evidence that reactivation of a memory returns it to a labile state, initiating a restabilization process termed reconsolidation, which allows for updating of the memory. In this study we investigated reactivation-dependent updating using a new positively motivated spatial task in rodents that was designed specifically to model a human list-learning paradigm. On Day 1, rats were trained to run to three feeders (List 1) for rewards. On Day 2, rats were trained to run to three different feeders (List 2) in either the same (Reminder condition) or a different (No Reminder condition) experimental context than on Day 1. On Day 3, rats were cued to recall List 1. Rats in the Reminder condition made significantly more visits to List 2 feeders (intrusions) during List 1 recall than rats in the No Reminder condition, indicating that the reminder triggered reactivation and allowed integration of List 2 items into List 1 . This reminder effect was selective for the reactivated List 1 memory, as no intrusions occurred when List 2 was recalled on Day 3. No intrusions occurred when retrieval took place in a different context from the one used at encoding, indicating that the expression of the updated memory is dependent upon the retrieval context. Finally, the level of intrusions was highest when retrieval took place immediately after List 2 learning, and generally declined when retrieval occurred 1-4 h later, indicating that the List 2 memory competed with short-term retrieval of List 1 . These results demonstrate the dynamic nature of memory over time and the impact of environmental context at different stages of memory processing.
\end{abstract}

Memories do not remain stable once acquired but, rather, change dynamically over one's lifetime. There is now strong evidence that reactivation of a memory (remembering) returns it to a labile state for a time, initiating a restabilization process that can allow for updating of the memory. This restabilization process, termed "reconsolidation," has been found to occur across a wide range of species and memory paradigms (Tronson and Taylor 2007; Nader and Einarsson 2010).

There is evidence in both humans and animals that new information available when a memory is reactivated can modify that memory as a consequence of reconsolidation. In a human procedural memory study, brief rehearsal of a consolidated fingertapping sequence prior to learning a new sequence resulted in impairment of the original memory when subjects were tested $24 \mathrm{~h}$ later (Walker et al. 2003). Critically, no impairment was observed if the original sequence was not rehearsed prior to learning the new sequence, indicating that reactivation affected the memory for the original sequence in such a way that subsequent learning could disrupt it.

It has recently been demonstrated in humans that reconsolidation can also be a constructive process, serving to update memories with new information (Hupbach et al. 2007). In this study, participants learned a list (List 1) of 20 objects on Day 1. On Day 2, the Reminder group was asked to recall the general procedure (but not the actual list) from Day 1 (the "reminder question"), and then learned a second list (List 2) of objects in the same room and with the same experimenter as on Day 1. The No Reminder group was not asked the reminder question, and learned the second list of objects in a different room with a different experimenter than on Day 1. On Day 3, in the same room and

\section{${ }^{4}$ Corresponding author.}

E-mail fellous@email.arizona.edu.

Article is online at http://www.learnmem.org/cgi/doi/10.1101/Im.023408.111. with the same experimenter as on Day 1, both groups were asked to recall the objects from Day 1 . The results showed that the Reminder group mistakenly recalled items from Day 2 (intrusions), whereas the No Reminder group did not. It was subsequently shown that the effective manipulation was the spatial context (the room) more so than the experimenter or the reminder question (or the combination of the two) (Hupbach et al. 2008). Recent imaging work has shown that the hippocampus is involved in the retrieval of spatial contexts in humans (Hoscheidt et al. 2010). These results suggest that the spatial context served to reactivate and, hence, destabilize the memory of List 1 , allowing for List 2 items to become integrated into the memory. As such, the spatial context is an important determinant of the dynamics of episodic memory (Nadel 2008).

In animals, most reconsolidation research has involved disrupting reconsolidation. Many of these studies used aversively motivated paradigms (Anokhin et al. 2002; Pedreira et al. 2002; Eisenberg et al. 2003; Debiec and LeDoux 2004; Gruest et al. 2004; Duvarci and Nader 2004; Runyan and Dash 2005). For example, bilateral infusions of the protein synthesis inhibitor (PSI) anisomycin into the lateral and basal nuclei of the amygdala following the reactivation of a consolidated fear memory significantly reduced the fear response (freezing) tested $24 \mathrm{~h}$ later in rats (Nader et al. 2000). Some appetitively motivated paradigms such as Pavlovian conditioning, conditioned place preference, and incentive learning have also been used to demonstrate the disruption of reconsolidation with pharmacological agents (Lee et al. 2005; Miller and Marshall 2005; Wang et al. 2005; Milekic et al. 2006; Lee and Everitt 2008; Milton et al. 2008; Valjent et al. 2006). For example, injection of the noncompetitive NMDA receptor antagonist, MK-801, following a retrieval trial of a well-learned appetitive spatial task (eight-arm radial maze task) impaired performance when rats were tested the next day (Przybyslawski and Sara 1997). These animal studies provide 
evidence that reconsolidation is necessary to restabilize a reactivated memory, such that the memory is maintained. None of these studies incorporated the effect of a new memory on an older one.

Some recent animal studies have focused on the role of reconsolidation in updating or integrating new information into memories rather than simply maintaining the memory. For example, post-retrieval injections of anisomycin into the hippocampus of rats trained on spatial tasks in the water maze resulted in subsequent memory impairment only when encoding of new information took place during retrieval (Morris et al. 2006). This study and others (Rossato et al. 2006; Rodriguez-Ortiz et al. 2008) have shown that the hippocampus is critical for updating spatial memories. Taste recognition (Rodriguez-Ortiz et al. 2005) and object recognition (Rossato et al. 2007) paradigms have also found evidence that reconsolidation is involved in updating memories with new information.

To date, the role of reconsolidation in updating spatial memories has been studied using water-maze tasks, which necessarily involve some level of stress. Given the uncertain impact of stress on memory and memory dynamics, it is important to assess the updating of spatial memories using an appetitively motivated paradigm that lacks this component of stress. In order to test spatial memory updating under relatively stress-free conditions, we designed a new positively motivated task equivalent to that of Hupbach et al. (2007) in humans (see Fig. 1 for the experimental design). Because of the importance of spatial context, we used the spatial context as a reminder to reactivate memory. Our hypothesis was that rats that are reminded of a previously consolidated spatial list of feeders while learning a new list of feeders would show integration of the new information during recall of the orig-
A

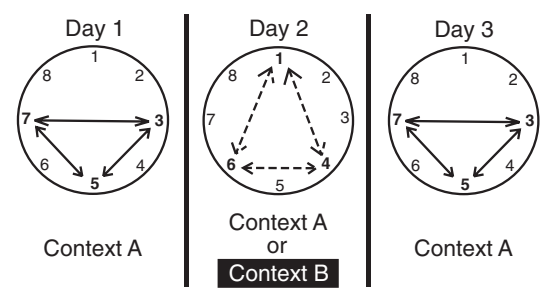

C

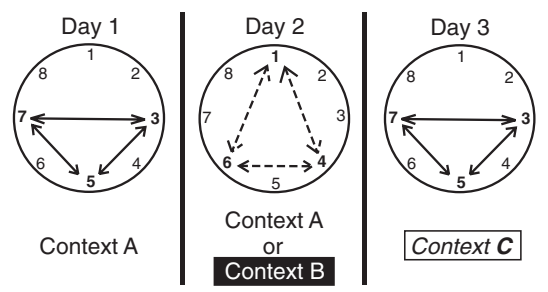

B

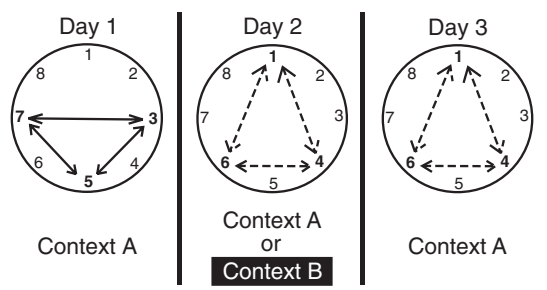

D
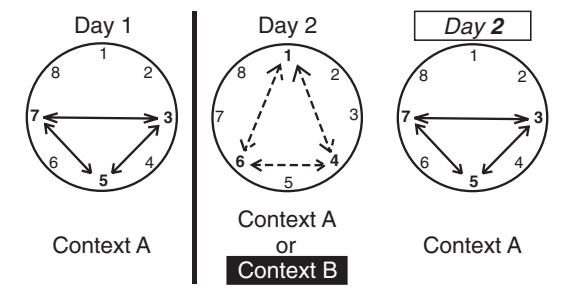

Figure 1. Experimental designs. (A) Experiment 1. Rats learned List 1 on Day 1 in Context A. Rats learned List 2 on Day 2 in either Context A (Reminder group) or Context B (No Reminder group). Rats were cued to recall List 1 in Context A on Day 3. (B) Experiment 2. Rats learned List 1 on Day 1 in Context A. Rats learned List 2 on Day 2 in either Context A (Reminder group) or Context B (No Reminder group). Rats were cued to recall List 2 in Context A on Day 3. (C) Experiment 3. Rats learned List 1 on Day 1 in Context A. Rats learned List 2 on Day 2 in either Context A (Reminder group) or Context B (No Reminder group). Rats were cued to recall List 1 in Context C on Day 3. (D) Experiment 4. Rats learned List 1 on Day 1 in Context A. Rats learned List 2 on Day 2 in either Context A (Reminder group) or Context B (No Reminder group). Rats were cued to recall List 1 in Context A on Day 2 either 15 min, $1 \mathrm{~h}, 2 \mathrm{~h}$, or $4 \mathrm{~h}$ following training for List 2. inal list, whereas rats that are not reminded would not show inteResults

Our study was designed to closely model the study performed in humans (Hupbach et al. 2007). In the first experiment (Experiment 1, study in rats by assessing the extent to which the contextual cues previously stored memory and make it susceptible to modifica tion. On Day 3, when animals were prompted to recall the list of feeders learned on Day 1 (List 1), animals that learned the second list of feeders on Day 2 (List 2) in a different context than List items (Fig. 2A, No Reminder, white bar) and could recall List 1 high accuracy (Fig. 2A, No Reminder, black bar). We then (Reminder condition). Because the context was identical during the learning of List 1 and List 2, it acted as a reminder of List 1. In this condition, the recall of List 1 featured a significant numor List 1 without it being explicitly recalled on Day 2 when Lis they learned. As a control, we ran animals in a condition in which pseudo-randomly chosen set of feeders (List 2) interfered with the retrieval of List 1 . The animals did not show any intrusions in this condition (Fig. 2A, Interference Control).

Statistically, there was no main effect of group on the cued recall of List 1 $\left(F_{(2,28)}=0.26\right)$, indicating that learning List 2 did not interfere with the ability of the rats to recall List 1 . However, a main effect of group was found for intrusions $\left(F_{(2,28)}=13.58, P<0.001\right)$. Post hoc comparisons (Tukey's HSD) revealed that there was no difference between the Interference Control and No Reminder groups in the percent of intrusions made $(P>0.05)$. However, the Reminder group was significantly more likely to show intrusions than both the No Reminder group $(P<0.001)$ and the Interference Control group $(P<0.001)$.

It is possible that the intrusions observed in the Reminder group are not due to reactivation and modification of the memory, but rather to the inability of the rats to distinguish between List 1 and List 2 . As both lists were learned in the same context, perhaps the Reminder group simply visited all feeders associated with that context. If this were the case, then we would expect that the Reminder group would also show more intrusions from List 1 into List 2 than the No Reminder group. Experiment 2 tested this possibility.

Results of Experiment 2 (Fig. 3A) illustrate that there was no difference between groups in the percentage of correct visits made to List 2 feeders during cued recall of List $2(t=1.03, P>0.05)$. There was also no difference between 
A

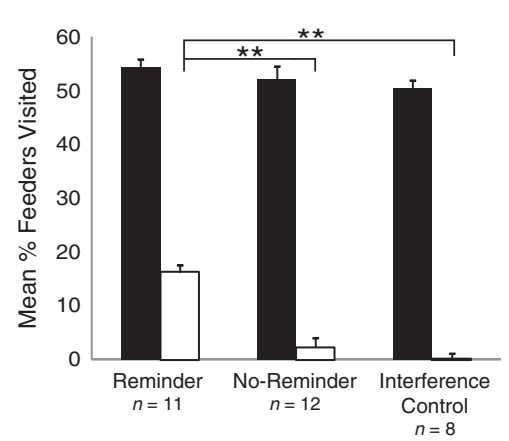

B

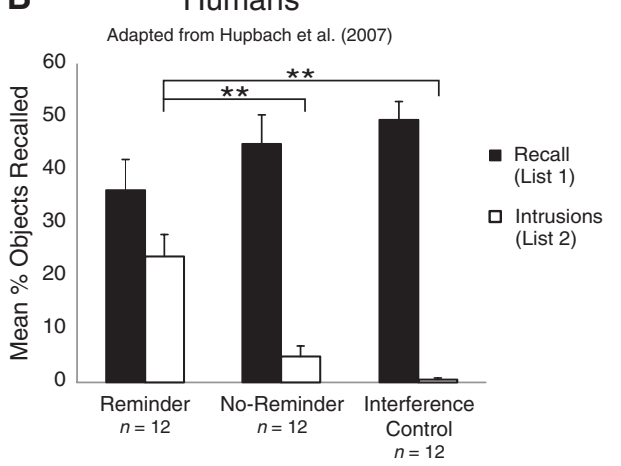

Figure 2. Experiment 1. (A) Mean percentage of feeders visited belonging to List 1 and List 2 during recall on Day 3 in the rat study. Error bars represent standard errors of means. Fourteen rats were used to obtain these data. (B) Mean percentage of objects correctly and falsely recalled on Day 3 in the Reminder, the No Reminder, and the Interference Control groups in the human study. Error bars represent standard errors of means. Note: Participants were asked to recall objects from List 1 . Objects that were falsely recalled from List 2 are labeled as Intrusions. Twelve subjects participated in each group (adapted from Hupach et al. 2007).

the percentage of List 2 feeder visits made in this experiment and the percentage of List 1 feeder visits made in Experiment 1 for either group (Reminder: $t=0.64$; No Reminder: $t=-0.18 ; P>0.05$ for both comparisons). In terms of intrusions, there was no difference between groups in the percentage of incorrect visits made to List 1 feeders $(t=0.25, P>0.05)$. Also, the Reminder group in this experiment made significantly fewer intrusions (List 1 into List 2) than did the Reminder group in Experiment 1 (List 2 into List 1) $(t=-4.85, P<0.001)$, whereas the No Reminder group in this experiment showed the same percentage (very low) of intrusions as did the No Reminder group in Experiment $1(t=0.23$, $P>0.05)$. These results are consistent with those found in the human study (Fig. 3B) and indicate that the reminder effect occurred specifically in the reactivated memory (List 1 ).

Memory retrieval is generally better when it takes place in the same context in which the memory was encoded. Although the rats are being cued to recall List 1 in Experiment 1, perhaps the Reminder group intrudes more List 2 feeders than the No Reminder group because the encoding and retrieval contexts for List 2 were consistent for the former group and inconsistent for the latter group. Therefore, in Experiment 3, we tested the reminder effect when retrieval took place in a neutral context, i.e., one in which neither list was encoded. Results are shown in Figure 4A. As in Experiment 1, there was no difference between groups in the percentage of correct visits made to List 1 feeders during recall of List $1(t=1.08, P>0.05)$. Additionally, there was no difference between the percentages of List 1 feeder visits made in this experiment and Experiment 1 for either group (Reminder: $t=-0.21$; No Reminder: $t=-0.72 ; P>0.05$ for both comparisons), indicating that the retrieval context did not affect recall of List 1. Unlike in Experiment 1, however, there was no difference between the Reminder and No Reminder groups in the percentage of intrusions made ( $t=-1.14, P>0.05)$ when recall took place in a different context. This result indicates that the reminder effect does not generalize to a neutral retrieval con-
A

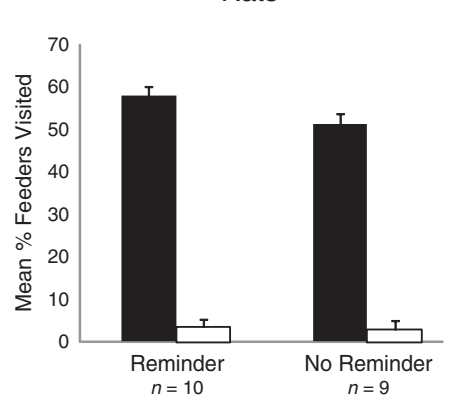

text in our study. Interestingly, a bimodal distribution was observed in the human study, with one half of the participants expressing intrusions in a neutral retrieval context (Fig. 4B).

If the reminder effect was the result of a time-dependent reconsolidation process, then there should be no effect when memory for List 1 is tested immediately after learning List 2 . However, the effect should emerge at a later recall test. In Experiment 4, we investigated the time course of the reminder effect, testing recall of List 1 immediately, $1 \mathrm{~h}, 2 \mathrm{~h}$, or $4 \mathrm{~h}$ (different groups at each time point) following List 2 learning. Results of Experiment 4 (Fig. 5A) show that there is no difference between groups in the percentage of correct visits made to List 1 feeders during cued recall of List 1 immediately following List 2 training $\left(F_{(2,27)}=\right.$ $0.50)$. However, there was a main effect of group on percent intrusions $\left(F_{(2,27)}=\right.$ $56.31, P<0.001)$. Compared with the Interference Control group, both the Reminder $(P<0.001)$ and No Reminder $(P<0.001)$ groups showed more intrusions. The Reminder group also showed significantly more intrusions than the No Reminder group $(P=$ 0.001). As Figure 5C illustrates, the intrusion levels for both groups generally declined with increasing retrieval intervals, with the Reminder group consistently making more intrusions than the No Reminder group at each time point $(1 \mathrm{~h}: t=3.26 ; 2 \mathrm{~h}: t=$ 5.19; 4 h: $t=2.38 ; P<0.05$ for all time points). As reconsolidation typically does not affect short-term memory, these results suggest that some form of short-term interference is causing intrusions at these time points.

\section{Discussion}

In Experiment 1, we found that rats recalled List 1 feeders equally well regardless of whether they learned List 2 in the original or new context, or whether they learned List 2 at all. This finding indicates that learning the second list did not interfere with the memory for the original list, and is in accord with a model of hippocampal learning that shows little interference of a newly learned list on a previously learned one (O'Reilly 2005). However, we found

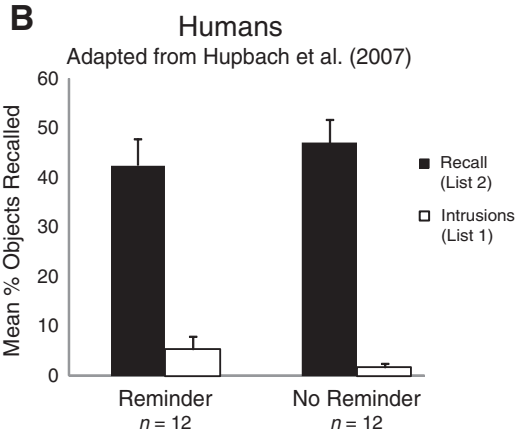

Figure 3. Experiment 2. (A) Mean percentage of feeders visited belonging to List 1 and List 2 during recall of List 2 on Day 3 in the rat study. Error bars represent standard errors of means. Eleven rats were used to obtain these data. (B) Mean percentage of objects correctly and falsely recalled from List 2 on Day 3 in the Reminder and the No Reminder groups in the human study. Error bars represent standard errors of means. Note: Participants were asked to recall objects from List 2. Objects that were falsely recalled from List 1 are labeled as Intrusions (adapted from Hupbach et al. 2007). 
A

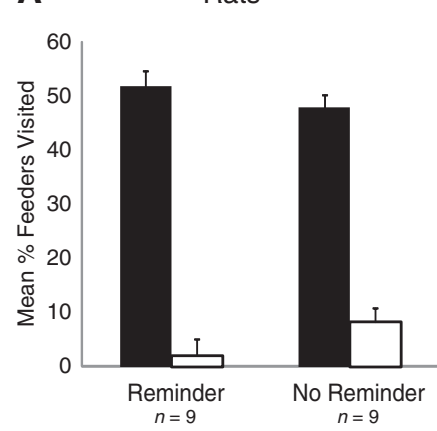

B

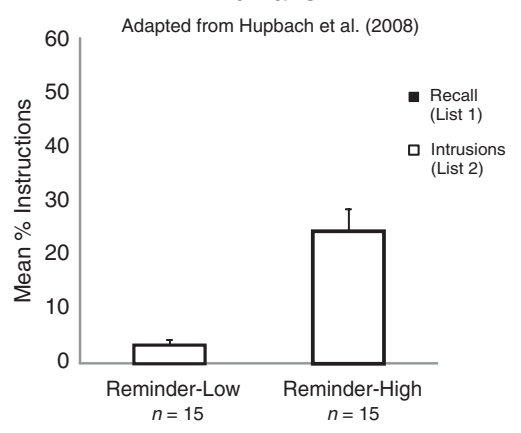

Figure 4. Experiment 3. (A) Mean percentage of feeders visited belonging to List 1 and List 2 during recall of List 1 on Day 3 in the rat study. Error bars represent standard errors of means. Nine rats were used to obtain these data. $(B)$ Mean percentage of objects falsely recalled from List 2 on Day 3 in the low-Intrusion and high-Intrusion Reminder groups resulting from a median split performed on the number of Intrusions in the human study. Error bars represent standard errors of means. Note: Participants were asked to recall objects from List 1. Objects that were falsely recalled from List 2 are labeled as Intrusions. Fifteen subjects participated in each group (adapted from Hupach et al. 2008).

that the Reminder group showed significantly more intrusions than the No Reminder group. Memory for List 2 was more often expressed during List 1 recall when List 2 was learned in the same context as List 1 . As both groups learned and consolidated List 2 , it seems unlikely that the intrusions would be caused by a consolidation effect. This result is consistent with previous studies showing that re-exposure to the experimental context is sufficient to induce reconsolidation in humans (Hupbach et al. 2008) and rodents (Artinian et al. 2007). Therefore, it seems that re-entering an environment triggers reactivation and reconsolidation of the List 1 memory, resulting in integration or linking of newly learned List 2 items into the original memory.

The results of Experiment 1 are compatible with that of Hupbach et al. (2007) (Fig. 2B) with a few differences. First, the mean percent of correct items recalled in rats was similar across the three conditions in the rats. In the human studies, the correct recall of the Reminder group was somewhat smaller than that of the No Reminder group, which, in turn, was smaller than that of the Interference Control group (2B, black bars). These differences were not statistically significant in the human experiments, but indicated a possible trend. Second, whereas the rat Interference Control group is very similar to the human Interference Control group in both percent correct recall and percent intrusions, the rat Reminder and No Reminder groups show higher percent correct recall and lower percent intrusions than the corresponding human groups. These differences may be due to the fact that correct recalls in our experiment were partially reinforced to keep the animals working, whereas there was no overt reinforcement during recall in the human experiment. Reinforcement may have led the rats to show less interference than the humans during recall of List 1, resulting in higher percent correct items (feeders) and lower percent intrusions in the rat Reminder and No Reminder groups.

These results show that our procedure can reproduce the main result of the human study, namely, that a reminder has the effect of making memory labile and subject to intrusions, even if the memory is not explicitly recalled.

An alternative explanation for this result is that, because both lists were learned in the same context, the Reminder group cannot distinguish between the two lists and simply visits all feeders that are associated with that context. If this were the case, then we would expect that the Reminder group would also show more intrusions from List 1 into List 2 than the No Reminder group. groups, though the encoding and retriev-

The results of Experiment 2 indicate that this was not the case, because the two groups did not differ in the percent of List 1 intrusions during recall of List 2. Additionally, the Reminder group of Experiment 2 showed significantly fewer intrusions (from List 1 into List 2) than did the Reminder group of Experiment 1 (from List 2 into List 1). This suggests that the Reminder group can distinguish between List 1 and List 2, and that the memory has temporal specificity in that intrusions occur from List 2 into List 1, but not vice-versa. This result is consistent with that found by Hupbach et al. (2007).

Memory retrieval is generally better when it takes place in the same context in which the memory was encoded. However, we found in Experiment 2 that recall of List 2 was comparable between the Reminder and No Reminder al contexts were consistent only for the Reminder group. Furthermore, we found in Experiment 3 that both groups recalled List 1 just as well in a neutral context (one in which neither list was encoded) as they did in Experiment 1, when the retrieval context matched the encoding context. Therefore, although the rats must use the contextual cues to navigate to the correct feeders during retrieval, it seems that they do not use the context as an indicator of which list to recall, instead most likely relying on the more salient light/reward cues.

Although the retrieval context does not influence the recall of the cued list in our paradigm, it could influence intrusions from the non-cued list. Indeed, we found in Experiment 3 that neither the Reminder nor the No Reminder group significantly intruded List 2 feeders during List 1 recall, with the Reminder group showing significantly fewer intrusions than in Experiment 1. Therefore, it seems that the expression of the updated memory is bound to the context in which it was encoded in our paradigm. Interestingly, when Hupbach et al. (2008) tested recall in a neutral context, they found a bimodal distribution, with only half of the human subjects showing intrusions. The performance of the rats is comparable to the subgroup that did not show intrusions in the neutral context. One possible explanation for the difference between the performance of the rats and the human subgroup that did show intrusions is that the rats rely more on automatic, cue-driven retrieval processes, whereas humans rely more on controlled, internally driven retrieval processes. Therefore, retrieval in rats would be more vulnerable to changes in contextual cues than retrieval in humans.

Previous studies have shown that the post-retrieval time window during which reconsolidation can be manipulated is very brief, indicating that some level of restabilization occurs shortly following reactivation. However, most studies do not see the behavioral effects of the manipulation until at least 3-4 h later, indicating that the manipulation does not affect short-term memory. However, in Experiment 4, we found that the reminder effect was present in short-term memory. Both the Reminder and No Reminder groups showed high levels of intrusions 15 min after List 2 learning. These levels dropped significantly when retrieval took place $1 \mathrm{~h}$ following List 2 learning, and remained roughly the same at the 2- and 4-h retrieval time points. Also, the Reminder group consistently showed higher levels of intrusions than the No Reminder group. The fact that the intrusion levels of both groups started high and generally decreased with increasing retrieval 
A

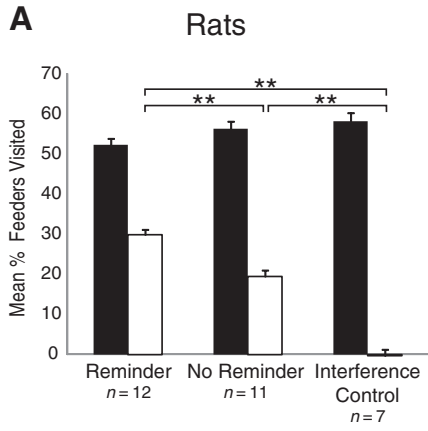

B
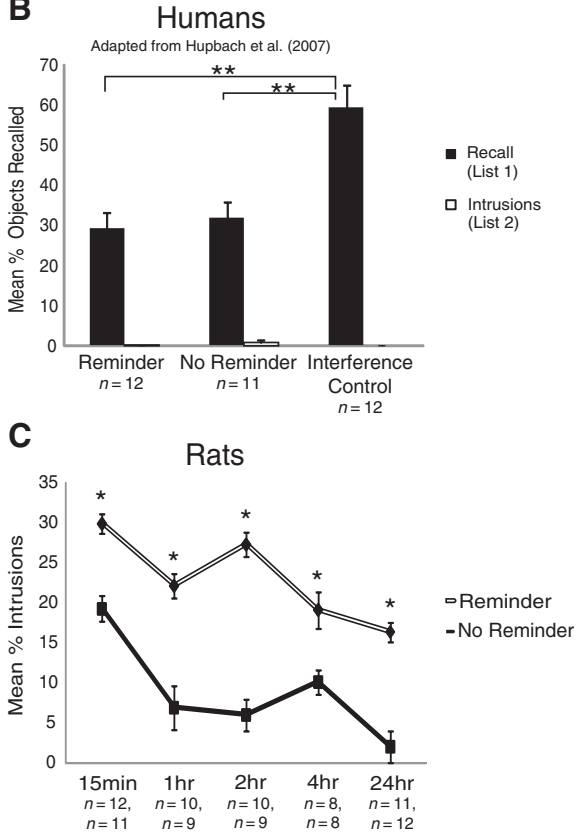

Figure 5. Experiment 4. $(A)$ Mean percentage of feeders visited from List 1 and List 2 during recall of List 1 on Day 2 in the rat study. Error bars represent standard errors of means. Fifteen rats were used to obtain these data. (B) Mean percentage of objects correctly and falsely recalled on Day 2 in the Reminder, No Reminder, and Interference Control groups in the human study. Error bars represent standard errors of means. Note: Participants were asked to recall objects from List 1. Objects that were falsely recalled from List 2 are labeled as Intrusions (adapted from Hupbach et al. 2007). (C) Mean percentage of Intrusions made during recall of List 1 either 15 min, 1 h, 2 h, 4 h, or 24 h following List 2 training in the Reminder and No Reminder groups. Error bars represent standard errors of means. Sixteen rats were used to obtain these data.

intervals suggests that the intrusions at these time points are due largely to competition between the two lists at the time of retrieval rather than to reconsolidation. As the Reminder group learned List 2 in the same context as List 1 retrieval, the competition at retrieval was stronger, resulting in higher levels of intrusions in the Reminder group than the No Reminder group.

Previous animal studies of reconsolidation that show intact post-reactivation short-term memory (PR-STM) typically look at memory for one learning episode. Here, we have investigated the effect of a second, similar learning episode on a previous learning episode. Therefore, it may be a limitation of this paradigm that we cannot assess PR-STM without seeing interference from the second episode. However, the effects of List 2 on the retrieval of List 1 would be expected to be strongest immediately following learning, when the List 2 memory is most active, and to decrease with time, perhaps as the memory is consolidated. This pattern is indeed what we observe. As the memory is assumed to be stable after $24 \mathrm{~h}$, it should no longer compete with the retrieval of List 1. At this time point, the level of intrusions for the No Reminder group has fallen to zero, but the intrusions are still high for the Reminder group. Therefore, reconsolidation is the most likely cause of intrusions in Experiment 1. However, as we would expect the List 2 memory to be reactivated by the List 1 retrieval context for the Reminder group (just as List 1 is reactivated on Day 2), we cannot rule out the possibility that this reactivation led to the intrusions.

Hupbach et al. (2007) found no intrusions when humans recalled an original list of objects immediately after learning a second list (Fig. 5B). However, the recall of List 1 was impaired in both the Reminder and No Reminder groups compared with the Interference Control group, indicating that the human subjects were also prone to some short-term interference. As with Experiment 3, the discrepancy between the human and rat data is likely due to differences in retrieval processes. As rats are likely to rely on automatic processing during retrieval, they may be unable to suppress recently learned items, whereas humans can suppress newly learned items via a deliberate and controlled process. Indeed, humans have been found to show greater interference when recalling items on an automatic basis compared with a controlled one (Lustig et al. 2004).

Figure 6A summarizes the number of intrusions observed at different retrieval time points following List 2 learning across all experiments in this study (left) and in the human study (right). Figure $6 \mathrm{~B}$ portrays the number of intrusions that would be expected to occur with time following List 2 learning based on different potential underlying memory processes. In both rats and humans, retrieval competition would produce high levels of intrusions shortly following List 2 learning, and these levels would decrease with time after learning. Retrieval competition would be higher for the Reminder than No Reminder group, resulting in a higher level of intrusions, because the List 2 learning and List 1 retrieval contexts are the same. In contrast to retrieval competition, reconsolidation would produce no intrusions shortly following List 2 learning (before cellular stabilization mechanisms) in both humans and rats. Intrusions would increase with time after learning in the Reminder group, but remain low in the No Reminder group. When retrieval competition is high shortly following List 2 learning, cognitive control mechanisms would be recruited in humans to actively suppress intrusions. The active suppression would decrease as retrieval competition decreases with time after learning. Active suppression would not occur in rats, at least not in this paradigm. Therefore, we conclude that the intrusions observed in our study are due to a combination of retrieval competition and reconsolidation processes, whereas the intrusions observed by Hupbach et al. (2007) might reflect these two processes plus active suppression.

How might memory updating be achieved at the cellular network level? The hippocampus has been shown to be critical for reconsolidation of spatial memories (Morris et al. 2006; Rossato et al. 2006; Rodriguez-Ortiz et al. 2008). In our study, the Reminder group learned the first and second lists of feeders in the presence of the same configuration of contextual cues, whereas the No Reminder group learned the first and second lists in the presence of different configurations of cues. It is known that place cells in the hippocampus "remap" when the animal enters a different or altered environment (Muller and Kubie 1987). Therefore, for the Reminder group, the first and second lists would be linked to the same place map, whereas, for the No Reminder group, the two lists would be linked to different place maps. Thus, intrusions from the second list into the first could be the result of the lists being linked indirectly through the same place map.

Research is increasingly uncovering the dynamic and reconstructive nature of memory. Modeling a human list-learning paradigm, we were able to extend the main result to a spatial memory 
A
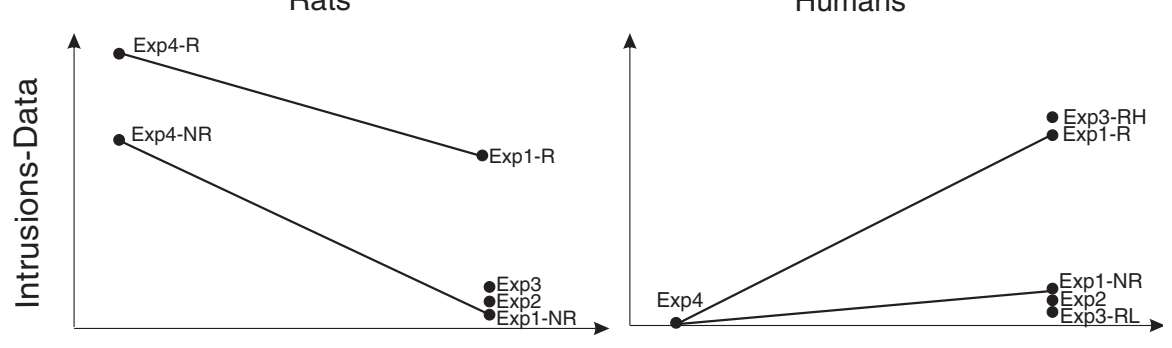

B

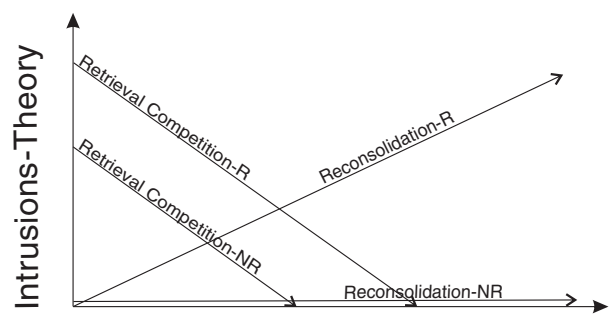

L2 Learning - L1 Recall Time Interval

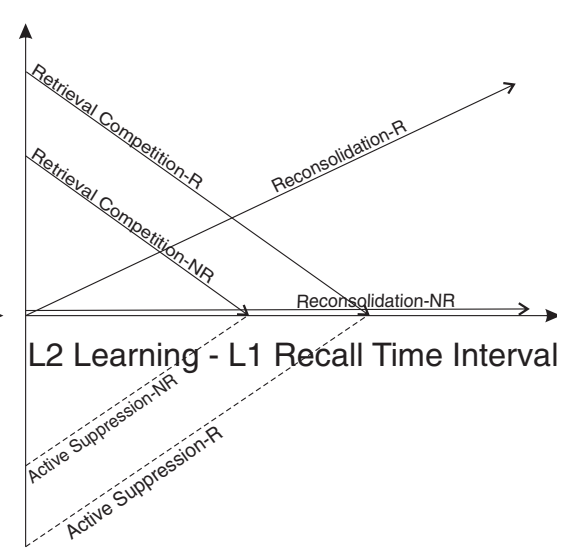

Figure 6. (A) Amount of Intrusions made during recall at different time points (across the different experiments) following learning of the intruding list in the rat (left) and human (right) studies. Conditions within each experiment are represented separately only if they are significantly different from one another. (B) Amount of Intrusions expected to occur with time following learning of the intruding list in rats (left) and humans (right) based on retrieval competition, active suppression, and reconsolidation. Only humans are expected to use active suppression. (Exp) Experiment, (R) reminder, (NR) no reminder, $(\mathrm{RH})$ reminder high, $(\mathrm{RL})$ reminder low.

task using an animal model. We found that reactivating a positively motivated spatial memory with contextual cues allowed it to be updated with new information. The ability to influence memory updating holds promise for various applications, including the treatment of post-traumatic stress disorder. Our findings highlight the dynamic nature of memory over time and the impact of environmental context at different stages of memory processing.

\section{Materials and Methods}

\section{Animals}

Sixteen adult (8-12 mo-old) male Brown Norway/Fisher 344 hybrid rats were housed in Plexiglas home cages, maintained on a reversed 24-h light/dark cycle, and food deprived to $85 \%$ of their ad libitum weight. Experiments were conducted in the dark phase. Rats were used across multiple experiments and conditions, with the order of conditions counterbalanced for each experiment. While all of the rats were reused, not all were available for the entire study, resulting in various numbers of animals across different experiments and conditions. The number of animals in each experiment and condition is noted in the figures. Different feeder lists and different combinations of sensory cues (contexts) were used for each different condition and experiment. In order to ensure that the animals did not have a preference for lists from previous experiments, they always received at least $1 \mathrm{~d}$ of random training between experiments, during which they were repeatedly cued to visit all eight feeders in random order. All procedures were in accordance with the animal care guidelines of the University of Arizona.

\section{Apparatus}

The apparatus used was an open-field circular arena that was $5 \mathrm{ft}$ in diameter and contained eight evenly spaced feeders around the periphery (Bower et al. 2005; Jones et al. 2007 abstract). The arena was lined by a 1 -ft tall wall at its periphery. Attached to each feeder was an LED light. Reward was sugar water $(0.2 \mathrm{~g} / \mathrm{mL})$. LED onset could be delayed to assess whether rats went to the feeders using memory alone. Rats were tracked by an overhead camera, and the feeders and lights were automatically controlled by a computer and custom-written software (Labview, National Instrument). Spatial context was manipulated as a combination of an odor (Renuzit air freshener, either apple cinnamon, sunny laundry, citrus sunburst, raspberry, tropical melon, after the rain, or vanilla), a floor texture (short carpet, bottom side of a carpet, or rubber mat), and one to two distal visual cues posted on the walls of the room ( 2 sq $\mathrm{ft}$ each, e.g., parallel lines or a triangle, high in contrast). A local cue was kept constant at the edge of the arena so that the rats could orient themselves on the table despite contextual changes.

\section{Training and test procedures: Experiment 1-the role of context as a reminder}

All animals were pretrained to randomly go to a blinking light to get a reward at the adjacent feeder (abstract in Jones et al. 2007). On Day 1 of the experiment, rats were cued by blinking lights to run to three predetermined randomly presented feeders (e.g. List $1=3,5,7)$ in the context of specific texture, odor, and distal visual cues (Fig. 1A, Context A). After they visited each feeder 50 times (150 rewards), the light cues were delayed by $15 \mathrm{sec}$, and the rats had to choose the feeders from memory. A rat typically travels between two feeders in $<6 \mathrm{sec}$, so the delay allowed enough time to reach the feeders with no cue. The delay was reset if the rat reached the feeder within $<15 \mathrm{sec}$, and the next light cue was activated with a new delay of $15 \mathrm{sec}$. The criterion was reached when the rats visited 15 correct feeders consecutively, with no more than two feeders being cued (i.e., the rats beat the light at least 13 out of 15 times). Once the criterion was reached, the rats received an additional 15 rewards with the light cues (no delays). On Day 2 of the experiment, the procedure was the same as for Day 1, except that a different list of feeders (e.g. List $2=1,4,6$ ) was used. There were three conditions on Day 2 . In the Reminder condition, rats learned List 2 in Context A. There was no explicit recall of List 1 on Day 2, so that context A was the only reminder of List 1 . In the No Reminder condition, rats learned List 2 in the context of different texture, odor, and distal visual cues (Context B). In the Interference Control condition, rats did not learn any list and remained in their home cages. This condition was included to control for any general effects that learning List 2 might have on the maintenance or retrieval of the List 1 memory. On Day 3, the rats were cued to recall List 1 in Context A (Fig. 1A). They received light cues and rewards only at List 1 feeders. The very first light cue was immediate in order to cue the rats as to which feeder list to recall, but the subsequent cues were delayed by 15 sec each. In order to keep the rats motivated while minimizing the level of reinforcement, a reward was given $50 \%$ of the time that the rats went to a correct feeder after having visited a different correct feeder, and $33 \%$ of the time after having visited an incorrect feeder. The test 
continued until performance reached the same criterion as during training.

\section{Training and test procedures: Experiment 2-the selectivity of the reminder effect}

The training and test procedures for Experiment 2 were exactly the same as for Experiment 1 except that the rats were cued to recall List 2 rather than List 1 on Day 3. Also, there was no Interference Control group in this experiment (Fig. 1B).

\section{Training and test procedures: Experiment 3-the role of the retrieval context}

The training and test procedures for Experiment 3 were exactly the same as for Experiment 1, except that the cued recall of List 1 took place in Context $\mathrm{C}$ rather than Context $\mathrm{A}$ on Day 3. There was no Interference Control group in this experiment (Fig. 1C).

\section{Training and test procedures: Experiment 4-the time course of the reminder effect}

The training and test procedures for Experiment 4 were exactly the same as for Experiment 1, except that the cued recall of List 1 took place either $15 \mathrm{~min}, 1 \mathrm{~h}, 2 \mathrm{~h}$, or $4 \mathrm{~h}$ (different groups at each time point) following List 2 training on Day 2 rather than on Day 3 (Fig. 1D).

\section{Data collection and analysis}

The feeder choices of the rat were recorded by both the experimenter and the computer program. Visits to each feeder type (List 1, List 2, and No List, the two remaining feeders that were neither part of List 1 nor List 2) are expressed as a percentage of the total number of feeders visited. These values have been normalized to account for the difference between the numbers of each feeder type. When analyzing recall, visits that were cued by the LEDs (when the rat failed to visit the correct feeder during the 15-sec delay) were not included. To calculate the percent correct recall and intrusions for each rat, the fraction of No-List feeders was subtracted from the fraction of List 1 and List 2 feeders, respectively, in order to control for baseline errors. (For the Interference Control group, a List 2 was chosen pseudo-randomly from the non-List 1 feeders.) To calculate percent recall and intrusions in Experiment 2, the fraction of No-List feeders was subtracted from the fraction of List 2 and List 1 feeders, respectively. Rats that failed to reach the learning criterion on Day 1 or Day 2 were excluded from the analysis (one data point [out of 12] from the Reminder condition and four [out of 16] from the No Reminder condition of Experiment 1 were excluded for this reason).

\section{Statistical analysis}

Differences between groups were analyzed using either one-way ANOVAs or unpaired two-tailed $t$-tests. When applicable, post hoc pair-wise comparisons were made using Tukey's HSD test. Significance levels were set to 0.05 .

\section{Acknowledgments}

This research was partially funded by NIA grant R36AG034230 (B.J.), the University of Arizona GIDP Neuroscience (B.J.), and NSF grant CRCNS 1010172 (J.-M.F.).

\section{References}

Anokhin KV, Tiunova AA, Rose SP. 2002. Reminder effects - reconsolidation or retrieval deficit? Pharmacological dissection with protein synthesis inhibitors following reminder for a passive-avoidance task in young chicks. Eur J Neurosci 15: 1759-1765.

Artinian J, De Jaeger X, Fellini L, de Saint Blanquat P, Roullet P. 2007. Reactivation with a simple exposure to the experimental environment is sufficient to induce reconsolidation requiring protein synthesis in the hippocampal CA3 region in mice. Hippocampus 17: $181-191$.

Bower MR, Euston DR, McNaughton BL. 2005. Sequential-contextdependent hippocampal activity is not necessary to learn sequences with repeated elements. J Neurosci 25: 1313-1323.

Debiec J, Ledoux JE. 2004. Disruption of reconsolidation but not consolidation of auditory fear conditioning by noradrenergic blockade in the amygdala. Neuroscience 129: 267-272.

Duvarci S, Nader K. 2004. Characterization of fear memory reconsolidation. J Neurosci 24: 9269-9275.

Eisenberg M, Kobilo T, Berman DE, Dudai Y. 2003. Stability of retrieved memory: Inverse correlation with trace dominance. Science $\mathbf{3 0 1}$ : 1102-1104.

Gruest N, Richer P, Hars B. 2004. Memory consolidation and reconsolidation in the rat pup require protein synthesis. J Neurosci 24: 10488-10492.

Hoscheidt SM, Nadel L, Payne J, Ryan L. 2010. Hippocampal activation during retrieval of spatial context from episodic and semantic memory. Behav Brain Res 212: 121-132.

Hupbach A, Gomez R, Hardt O, Nadel L. 2007. Reconsolidation of episodic memories: A subtle reminder triggers integration of new information. Learn Mem 14: 47-53.

Hupbach A, Hardt O, Gomez R, Nadel L. 2008. The dynamics of memory: Context-dependent updating. Learn Mem 15: 574-579.

Jones B, McClung A, Hupbach A, Hardt O, Gomez R, Nadel L, Fellous JM. 2007. Dynamics of sequence learning in rats: The influence of reminders and training by blocks. Society for Neuroscience, San Diego, CA (poster).

Lee JLC, Everitt BJ. 2008. Appetitive memory reconsolidation depends upon NMDA receptor-mediated neurotransmission. Neurobiol Learn Mem 90: 147-154.

Lee JLC, Di Ciano P, Thomas KL, Everitt BJ. 2005. Disrupting reconsolidation of drug memories reduces cocaine seeking behavior. Neuron 47: 795-801.

Lustig C, Konkel A, Jacoby LL. 2004. Which route to recovery? Controlled retrieval and accessibility bias in retroactive interference. Psychol Sci 15: $729-735$.

Milekic MH, Brown SD, Castellini C, Alberini CM. 2006. Persistent disruption of an established morphine conditioned place preference. J Neurosci 26: 3010-3020.

Miller CA, Marshall JF. 2005. Molecular substrates for retrieval and reconsolidation of cocaine-associated contextual memory. Neuron 47: $873-884$.

Milton AL, Lee JLC, Everitt BJ. 2008. Reconsolidation of appetitive memories for both natural and drug reinforcement is dependent on $\beta$-adrenergic receptors. Learn Mem 15: 88-92.

Morris RG, Inglis J, Ainge JA, Olverman HJ, Tulloch J, Dudai Y, Kelly PA. 2006. Memory reconsolidation: Sensitivity of spatial memory to inhibition of protein synthesis in dorsal hippocampus during encoding and retrieval. Neuron 50: 479-489.

Muller RU, Kubie JL. 1987. The effects of changes in the environment on the spatial firing of hippocampal complex-spike cells. J Neurosci 7: 1951-1968.

Nadel L. 2008. The hippocampus and context revisited. In Hippocampal place fields: Relevance to learning and memory (ed. SJY Mizumori), pp. 3-15. Oxford University Press, New York.

Nader K, Einarsson EO. 2010. Memory reconsolidation: An update. Ann N Y Acad Sci 1191: 27-41.

Nader K, Schafe GE, Le Doux JE. 2000. Fear memories require protein synthesis in the amygdala for reconsolidation after retrieval. Nature 406: $722-726$

O'Reilly RC. 2005. The division of labor between the neocortex and hippocampus. In Connectionist modeling in cognitive psychology (ed. $\mathrm{G}$ Houghton). Psychology Press, London, UK.

Pedreira ME, Perez-Cuesta LM, Maldonado H. 2002. Reactivation and reconsolidation of long-term memory in the crab Chasmagnathus: Protein synthesis requirement and mediation by NMDA-type glutamatergic receptors. J Neurosci 22: 8305-8311.

Przybyslawski J, Sara SJ. 1997. Reconsolidation of memory after its reactivation. Behav Brain Res 84: 241-246.

Rodriguez-Ortiz CJ, De la Cruz V, Gutierrez R, Bermudez-Rattoni F. 2005. Protein synthesis underlies post-retrieval memory consolidation to a restricted degree only when updated information is obtained. Learn Mem 12: $533-537$.

Rodriguez-Ortiz CJ, Garcia-DeLaTorre P, Benavidez E, Ballesteros MA, Bermudez-Rattoni F. 2008. Intrahippocampal anisomycin infusions disrupt previously consolidated spatial memory only when memory is updated. Neurobiol Learn Mem 89: 352-359.

Rossato JI, Bevilaqua LR, Medina JH, Izquierdo I, Cammarota M. 2006. Retrieval induces hippocampal-dependent reconsolidation of spatial memory. Learn Mem 13: 431-440. 
Rossato JI, Bevilaqua LR, Myskiw JC, Medina JH, Izquierdo I, Cammarota M. 2007. On the role of hippocampal protein synthesis in the consolidation and reconsolidation of object recognition memory. Learn Mem 14: 36-46.

Runyan JD, Dash PK. 2005. Inhibition of hippocampal protein synthesis following recall disrupts expression of episodic-like memory in trace conditioning. Hippocampus 15: 333-339.

Tronson NC, Taylor JR. 2007. Molecular mechanisms of memory reconsolidation. Nat Rev Neurosci 8: 262-275.

Valjent E, Corbille AG, Bertran-Gonzalez J, Herve D, Girault JA. 2006. Inhibition of ERK pathway or protein synthesis during reexposure to drugs of abuse erases previously learned place preference. Proc Natl Acad Sci 103: 2932-2937.

Walker MP, Brakefield T, Hobson JA, Stickgold R. 2003. Dissociable stages of human memory consolidation and reconsolidation. Nature 425: $616-620$.

Wang SH, Ostlund SB, Nader K, Balleine BW. 2005. Consolidation and reconsolidation of incentive learning in the amygdala. J Neurosci 25: $830-835$.

Received July 8, 2011; accepted in revised form January 10, 2012. 


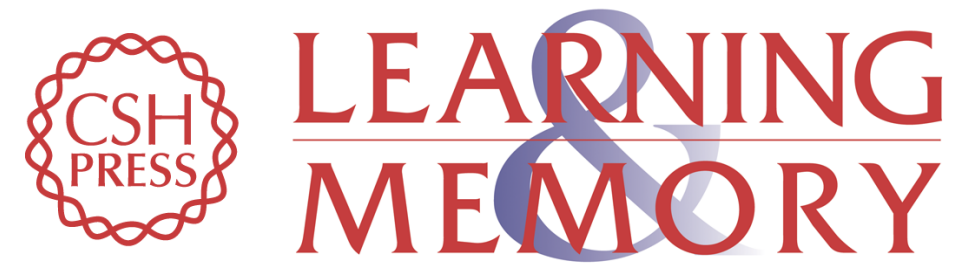

\section{Remaking memories: Reconsolidation updates positively motivated spatial memory in rats}

Bethany Jones, Elizabeth Bukoski, Lynn Nadel, et al.

Learn. Mem. 2012, 19:

Access the most recent version at doi:10.1101/Im.023408.111

References This article cites 31 articles, 15 of which can be accessed free at: http://learnmem.cshlp.org/content/19/3/91.full.html\#ref-list-1

License

Email Alerting Receive free email alerts when new articles cite this article - sign up in the box at the Service top right corner of the article or click here. 